\title{
Simulation and calculations of processing and transportation of hydrocarbon streams in spreadsheets
}

\author{
O. A. Omelchenko ${ }^{1}$, D. A. Rychkov ${ }^{1}$, V. V. Prytkov ${ }^{1}$, \\ D. E. Ukraintseva ${ }^{1}$, A. G. Kasperovich ${ }^{2}$ \& M. V. Ovsyankin ${ }^{2}$ \\ 'OOO "TyumenNIIgiprogaz", Russia \\ 2OOO "Gazprom pererabotka”, Russia
}

\begin{abstract}
Modern software packages for simulation of technological processing and transportation of hydrocarbon streams are generally not well suited for use by non-trained professionals due to their high functional content, complexity and high cost of operation. In response to engineering community needs, the authors developed their Microsoft Excel based tools for modeling technological processes and performing stand-alone calculations of component-fractional composition, physical and chemical properties, matter and component processing balances, as well as modeling thermal and hydraulic regimes of transport of hydrocarbon condensate streams in oil and gas fields. Model development and calculations in the package are implemented in the form of user-defined functions and functional procedures (macro-procedures). The number crunching procedures are programmed in CodeGear Delphi and compiled as a DLL library. The functionality of this library and macro-procedures in the Microsoft Excel environment is achieved via add-on files. The underlying computational model is based on known thermodynamic laws and correlations and the authors' original methods. In particular, rectification is simulated via a sigma-function, which generally characterizes the efficiency of mass transfer. The proposed tools are not intended to replace more sophisticated thermodynamic simulations but rather provide sufficient functionality and accuracy of calculations together with a short learning curve to a broad range of professionals familiar with the Microsoft Office environment.
\end{abstract}

Keywords: simulation of technological processing and transportation of hydrocarbon streams, software packages for simulation. 


\section{Introduction}

Currently there is a large range of products for common technological and thermodynamic simulations (technological modelling systems - TMS). These include Aspen HYSYS (AspenTech), GIBBS (OOO "Topenergobiznes") GazKondNeft (NTF "Termogaz") and others. All of these systems allow developing complex systems out of elementary parts and enable detailed technological calculations. They include numerous computational methodologies which must be specified before the user proceeds with calculations. However, there is often a need to address multivariate balance problems with simultaneous output and visual display of results for analysis that can be effectively implemented in Microsoft Excel. It is for this purpose that a modelling methodology has been developed for field preparation and processing of hydrocarbons based on configurable outputs and quality streams of thermodynamic and statistical selection (distribution) functions of the components (FSC). Based on this methodology, the authors created computational models of refineries and other hydrocarbon processing facilities.

\section{Concepts methodology}

A basic characteristic of hydrocarbon streams which is considered in the methodology is the component- fractional composition (CFC) - mass (percent) content of individual components to pentanes inclusive and more narrow $10-$ degree (at boiling point) fractions (pseudo- components). Each mass transfer unit (and even a large processing unit) is represented as a node of separation (NS) with one input stream and two output (products) flows: easy (relatively steam) flow and heavy (liquid) flow. Fig. 1 shows a technological example of a condensate stabilization unit (CSU) in the data stream model of two NSs.

The model development methodology is based on the representation of a technological field-preparation scheme in the form of a set of interrelated binary split nodes (including recycling) which simulate mass transfer devices. Here detailed calculations of mass transfer processes are replaced with calculations using generalized functions of component selection which characterize the efficiency of NS. FSC is a (percentage-based) relation of selection coefficients (SC) for components (fractions) of raw materials in the heavy output of the NS considered. Thus the SC component (fraction) is calculated by the formula:

$$
K_{i}^{L}=\frac{G_{i}^{L}}{G_{i}^{i n}} \cdot 100 \%
$$

where: $K_{i}^{L}$ is the liquid selection coefficient for the i-th element of CFC; $G_{i}^{L}$ is the content by weight (or mass) of liquid separation flow; $G_{i}^{i n}$ is the content by weight (or mass) of input (raw) flow in NS. 

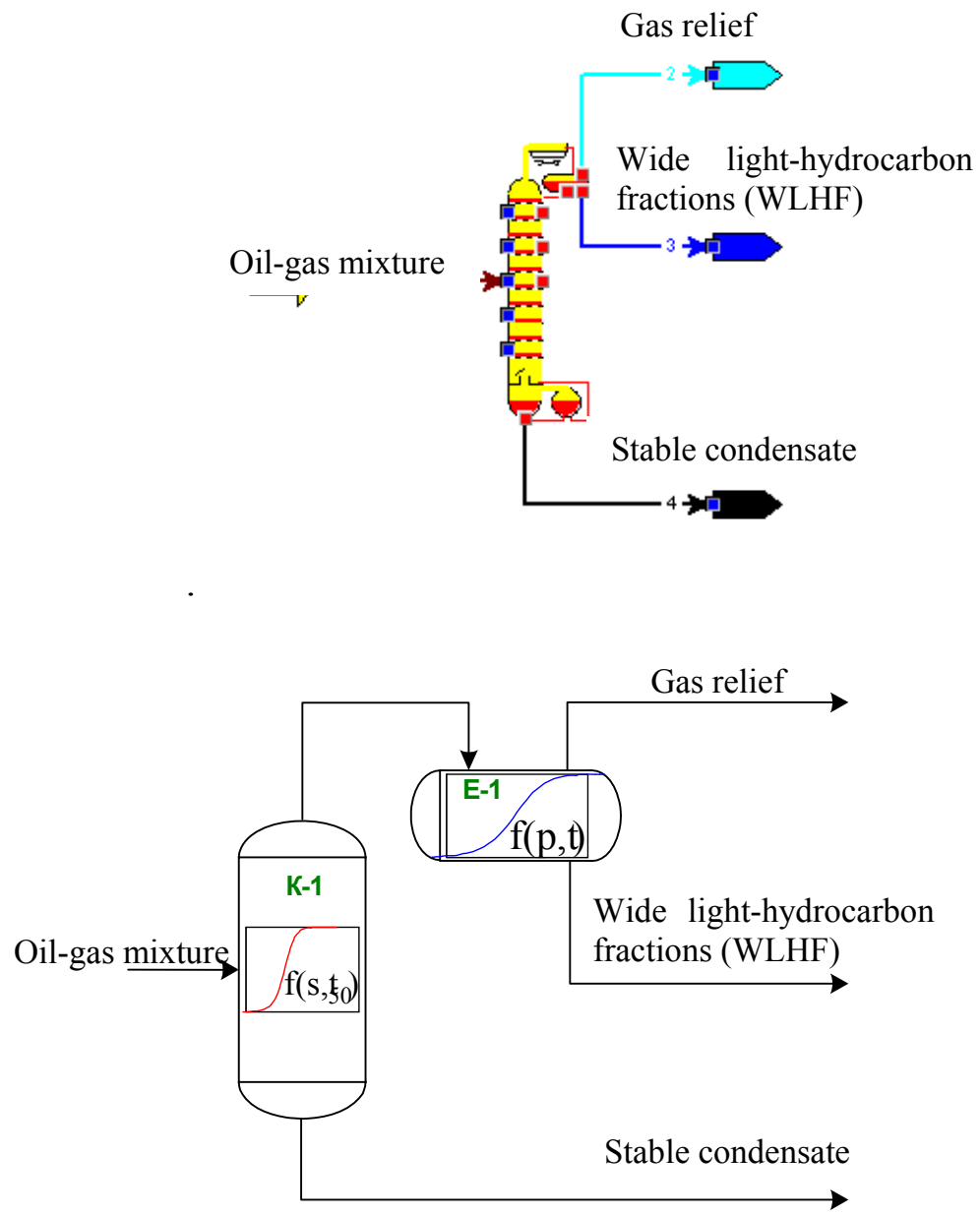

Figure 1: Conversion of a technological scheme of CSU into a stream model.

FSC different processes are shaped like a s- shaped curve (see Fig. 2).

The efficiency of mass transfer of NS depends on the curve slope, which in turn depends on technological parameters of a model element considered. For example, for a stabilization column the slope varies significantly depending on the reflux ratio and the number of theoretical plates (see Fig. 3)

When the temperature and pressure conditions of a technological apparatus change then the FSC of the corresponding NS move equidistantly with a good degree of certainty along the temperature axis (see Fig. 4).

To characterize this change of FSC we use the temperature of a key Tb50 component which is the boiling point of a hypothetical component uniformly distributed $(50 \%)$ between the products of a node of separation. Our model development experience suggests that the best way to get an equidistant "image" 
848 Energy Production and Management in the 21st Century, Vol. 2

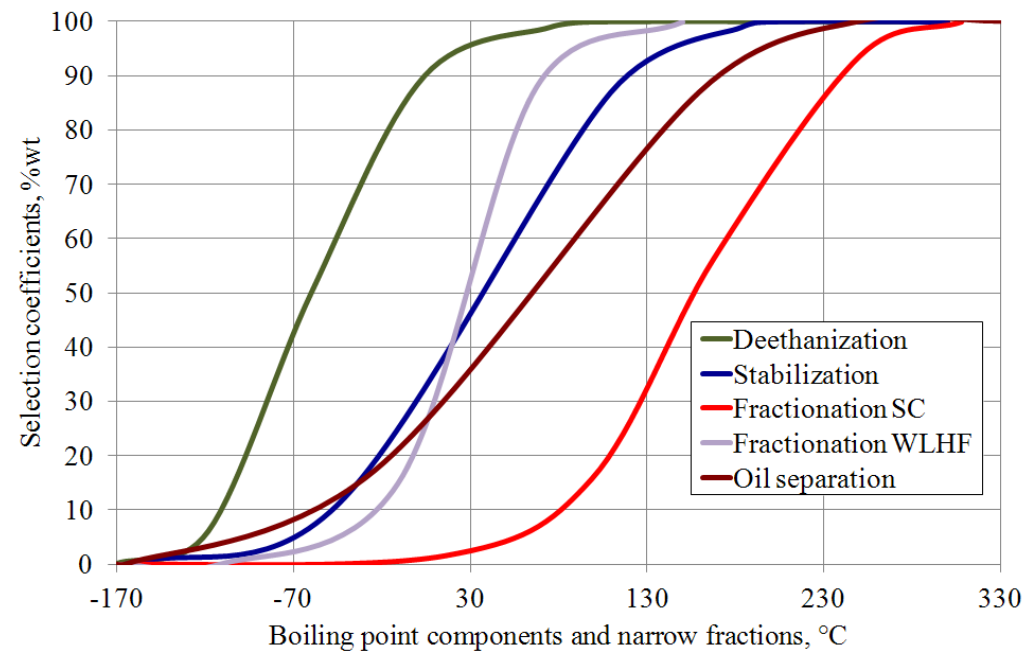

Figure 2: Features selection of components of different technological elements.

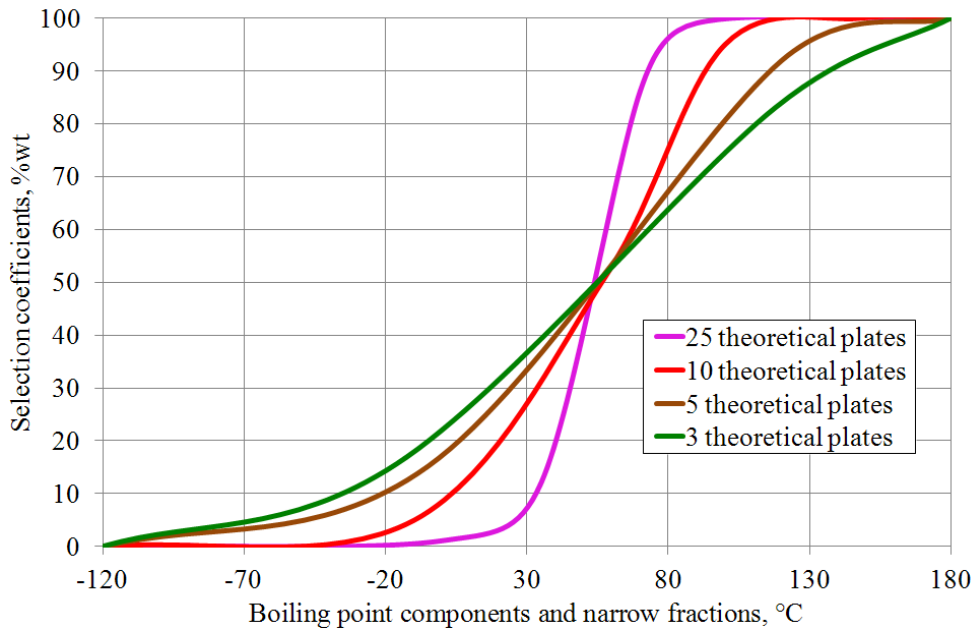

Figure 3: Component selection functions for the stabilization column for a reflux ratio of 1 and different numbers of theoretical plates. 


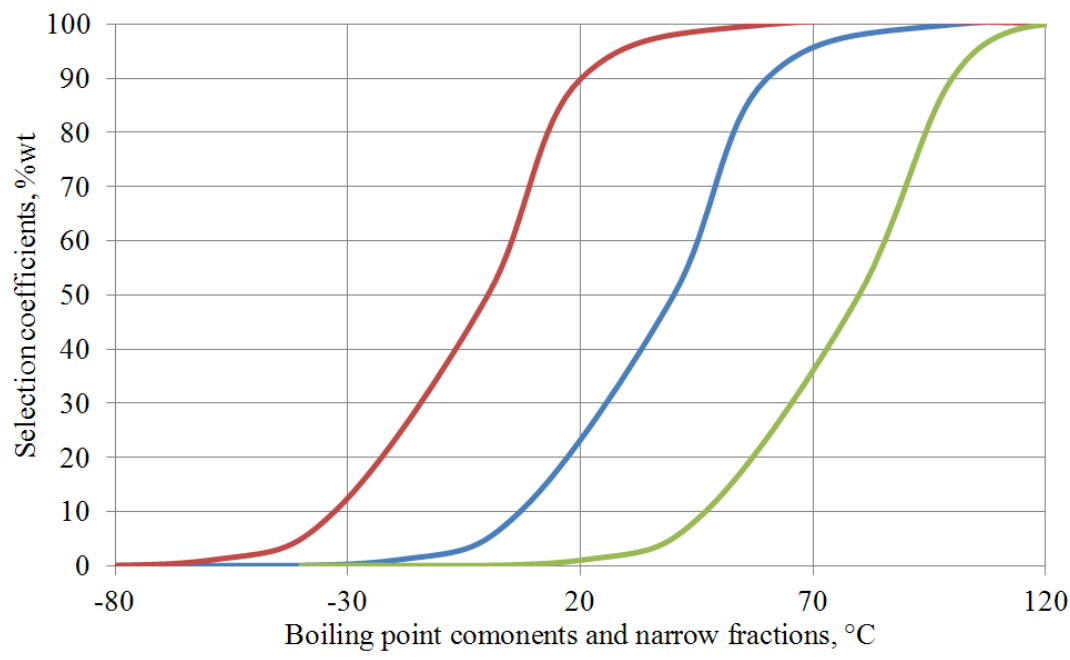

Figure 4: Change in the component selection functions of the stabilization column (10 theoretical plates, reflux ratio 1) with the temperature.

in the operating conditions of a technological NS is the closest analytical expression that describes the shape of the FSC. Such is a sigmoid function:

$$
K_{i}=100-\frac{100}{1+\exp \left[S_{i} \cdot\left(T b_{i}-T b_{50}\right)\right]}
$$

where: $K_{i}$ is the selection coefficient of i-th component in the liquid (heavy) product NS,\%; $S_{i}$ is the slope factor for an individual component (fraction); $T b_{i}$ is the boiling point of the i-th component, ${ }^{\circ} \mathrm{C}$.

Entering individual slope factors is logical, since FSC is not a smooth line, but rather a multi-joint unit with nodes placed at the points of components (fractions). From (2) we compute the slope factors:

$$
S_{i}=\frac{\ln \left[1 /\left(1-K_{i} / 100\right)-1\right]}{T b_{i}-T b_{50}}
$$

Evaluating these factors for various setups (for example, a distillation column, reflux ratios for various and different number of theoretical plates) is an important task. This process forms a so-called "unit passport". The formation of a passport may be a result of the processing of experimental structures of output products (retrospective calculations) or may be done by using the TMS for new modes (predictive calculations). When the mass transfer characteristics change then sigmoid profile is modified accordingly. This is achieved by using various 
methods of interpolation of array $\mathrm{Si}$ of several variables responsible for the mode of operation of the unit. For processing the experimental CFC different smoothing algorithms can be used, which are also incorporated into the methodology.

Based on the above, the following stages can be emphasized when working with each node of separation in the model:

1. Adaptation using retrospective experimental data, special research work, getting a passport of NS;

2. Solving the main problem of NS calculations, i.e. getting the material and component balance (MCB) for a specified mode of NS.

Let us dwell on the second stage. The problem of determining FSC for a desired mode is reduced to the iterative search for sigmoid slope factors that satisfy a given specification of one of the products of separation. This can be physical and chemical properties of the stream and quality/yield metrics of products in mass terms. This methodology includes a number of calculations used in specifications as parameters. One may also offer economic and other non-system indicators, which gives the potential to develop methodology.

The calculation itself is performed as follows. First, using methods of multicriteria interpolation of slope factor data one finds new sigmoid slope factors specific to a given mass transfer mode. Then using single parameter solution techniques (dichotomy or a combination of dichotomy and secant methods) - a key parameter Tb50 is found. By using the slope factors and Tb50 in each iteration one recovers FSC. The final solution is found by satisfying the accuracy required for given specifications of the NS.

In some cases, one may need to perform a specific task: the selection of thermobaric separation parameters (pressure and temperature) for a known value of either of them in order to achieve a given specification. In such ("thermodynamic") NSs the same methods are used but the estimated FSC are replaced by functions with SC numerically equal to the coefficients of thermodynamic equilibrium. As an equation of state the well-known cubic equation of Peng-Robinson is chosen. This method can be used for recovery of the raw stream of a separator based on a known composition of the liquid product and separation conditions (pressure, temperature).

\section{Software composition}

In the process of shaping this methodology the following interconnected tools have been created for developing simulation models:

1. Add-in files for Microsoft Excel. They contain screen setting forms for macro-procedures, macro-procedures implemented in language VBA; declarative references to functions and functionals whose code resides in a specially created DLL. There are also add-ins, which offer user-defined functions implemented in VBA (Visual Basic for Application).

2. A dynamic DLL library implemented in the programming environment CodeGear ${ }^{\text {TM }}$ 2007. This library implements functions and functionals whose 
declarations (and their link with Excel) are found in add-in files provided. A function computes a single result. The notion of "functional" assumes some processing, which results in ranges of data, and even more massive calculations on separately allocated sheets. A number of such complex functionals are used for adaptation and adjustment of models. For example, these functionals model differential condensation and balance of reservoirs. The practice of creating functionals is currently expanding. They are implemented directly in VBA for processing of reports, texts unloading of TMS, etc.

3. Xls program communication patterns and TMS. Such combined models provide more detailed calculations of hydrocarbon processing plants and transmit results to an xls model for analysis. After performing the necessary analysis and correction in the xls model through iterative exchange of data with TMS one can solve the problem of recovery of raw materials with a known composition of one of the products.

It should be emphasized that the methodology presented here does not rely on the conventional approach for building models out of elementary constituents. Models are set up using forms, custom menu, and interactive buttons. All settings are in the form of a certain structure or worksheet in a Excel workbook, from which the model gets parameters for nodes of separation and all phases of the computational process. To understand the process of construction of such models one needs good Microsoft Excel skills, technological expertise, familiarity with the contents of the toolkit. To facilitate the process of familiarization with the proposed technology, a number of documentation packages, test models and guidelines have been created.

\section{Overview of physical and chemical properties of built-in models and mathematical algorithms}

\subsection{Physical and chemical properties}

The basic concept of our methodology and most of the calculations used are given in [1]. In addition, the authors systematized documentation updates in the form of guidelines for "Gazprom pererabotka" ("Guidelines on the calculation of the composition, properties, modes of transport and processing of hydrocarbon streams balances condensate and oil and gas fields," MR -03- 2011, 28.12.2011, № 450).

To start performing calculations one needs to obtain information on physical and chemical properties of CFS in the right format (data ranges for Microsoft Excel): molecular weight, boiling point, caloric, critical parameters (pressure and temperature), acentric factor, characteristic volume (separate array for calculating the density of the procedure COSTALD). These data can be taken from reference sources, obtained experimentally or calculated using the features built into the software. In particular, there are functions for calculating critical properties (temperature and pressure), acentric factor, calorie and others for fractions. The known correlations [2] are used for this purpose. Models often 
consider mixing of flows with significantly different properties of fractional composition. The authors have created a functional for calculating properties of mixed flows with different mixing rules. To calculate the vapor - liquid equilibrium, the authors have taken as a basis the cubic equation of state (EOS) of Peng-Robinson [2-5]. It should be noted that in order to adapt various algorithms to experiment the authors have applied the most appropriate calculation of critical properties of fractions using their original solutions.

Thus, being based on the properties of the CFS described below, the software library implements the following properties of a hydrocarbon stream (including the assignment of thermobaric conditions): molecular weight, density, calorific value, viscosity, surface tension, pour point, cloud point, octane, cetane number of properties on the saturation line (initial boiling point, vapor pressure, dew point temperature, pressure dew point), gas and gas condensate ratio and others. Note that for the density of the liquid flow the Hankinson-Thompson (COSTALD) method was chosen [6]. The authors have modified algorithms for saturation line properties very extensively. The past experience has shown that using traditional methods (e.g., the Peng-Robinson EOS) frequently results in failures and the lack of reliable results. The authors approached this problem by using approximate interpolation techniques for the mole fraction of the liquid phase to the specified boundary phase transition (linear and spline interpolation). These results are more stable and adequate, giving greater confidence in the calculations.

\subsection{Models of experimental studies}

When determining FSC in NS one may use as specifications for the desired flow both physical and chemical properties and quality indicators derived by experimental methods. Accordingly, the authors have developed and implemented computational models of the most common experimental studies. Among them are: differential condensation, fractional distillation by Engler, pressure of saturated vapor pressure, methods of degassing volatile liquid hydrocarbons. We have created and are now improving a model for the evaluation of a gas condensate reservoir. In this model the material balance calculation is done by breaking down the reservoir into cells in which the pressure varies in accordance with the specified bottom hole pressure. In addition, the model accounts for removal of the liquid phase at certain maximum condensate saturation. Almost all existing models employ the algorithm for calculating vapor-liquid equilibria using the cubic Peng-Robinson equation. Models are constantly being improved in accordance with experimental data and current knowledge of the subject.

\subsection{Mathematical algorithms}

As described above, in the search for solutions at various FSC in both calculation-driven models and in the case of adaptation of experimental data, the authors use single-parameter and multi-parameter interpolation techniques. Among them are methods of linear interpolation, cubic spline interpolation, and 
Shepard's multi-parametric interpolation. To find solutions when obtaining FSC we use dichotomy and combinations of dichotomy and secants. In some cases, models use Microsoft Excel solutions. In order to process the composition data and handle calculation results, the authors introduce smoothing techniques (the Gauss window, local polynomial approximations, smoothing of the sigmoid).

\section{Development}

The authors believe that their methodology for calculating material and component balances of hydrocarbon streams on the basis of FSC has broad prospects. On the one hand, it can be used by different specialists (engineers, project and research scientists); on the other hand there is a real possibility of extending the algorithms to other fields (for example, entering the economic parameters of quality) and related industries (petrochemicals). We are currently in the process of developing technical documentation (guidelines, measurement techniques, standard organizations, etc) for this methodology. The development of computational models of experimental studies is a separate promising direction, since such studies are often time-consuming and costly. The authors find further development of the algorithm for assessment calculation of a gas condensate reservoir very interesting. Naturally, the authors do not claim their model to be very accurate. However, if the adopted model shows a good correlation with experiment and calculations from more detailed models (e.g., Eclipse - 300, Schlumberger), then this multivariate assessment methodology will be in demand. Therefore, the authors believe that the proposed methodology has a good potential for future development.

\section{Conclusion}

This report sets out the main provisions of the original methodology developed by authors for modeling technological processes, calculations of composition, physical and chemical properties, material and component balances of processing and thermal hydraulic modes of transport of hydrocarbon streams of gas, condensate and oil fields. The principles and means of the implementation in the public environment (Microsoft Excel spreadsheets) are also outlined. The proposed solutions allow a broad range of professionals (including those who do not have access to modern technological modeling systems) to perform quite complex, multi-step and multiple calculations. The extensive functionality and the user-friendly spreadsheet interface provide opportunities for the most diverse range of tasks, as well as application of similar tools in related fields.

\section{References}

[1] Kasperovich A.G., Magaril R.Z., Balance calculations in the design and planning of hydrocarbon processing gas condensate and oil and gas fields, [in Russian], "University Book House": Moscow, 2008. 
854 Energy Production and Management in the 21st Century, Vol. 2

[2] Brusilovsky A.I., Phase transformations in the development of oil and gas fields, [in Russian], Publishing house "Graal": Moscow, 2002.

[3] Brusilovsky A.I., Nugaeva A.N., Theory and practice study properties of natural hydrocarbon systems, [in Russian], "ZAO Askonline": Moscow, 2008.

[4] Reed R., Prausnits J., Sherwood T., Properties of gases and liquids, [in Russian], "Chemistry": Leningrad, 1982.

[5] Ueyles S., Phase equilibria in chemical engineering, [in Russian], "Mir": Moscow, 1989.

[6] Hankinson R.W., Coker T.A., Thomson G.H., Get accurate LNG densities with COSTALD. Hydrocarbon Process: United States, 62, pp. 207-208, 1982. 